\title{
Personalizing Drug Administration to Patients with Parkinson's Disease: Manipulating Sensor Generated Data in Android Environments
}

\author{
Klemen Bravhar \\ HSN, Kongsberg Institute of Engineering \\ klemen.bravhar@student.hsn.no
}

\author{
Radmila Juric \\ HSN, Kongsberg Institute of Engineering \\ rju@hbv.no
}

\begin{abstract}
This paper illustrates the application of mobile and wireless technologies for estimating the severity of Parkinson Disease symptoms, and performing a personalized drug administration to PD patients. The measurements of patient finger pressures on the screen of a smart phone, translated into analogue voltage and digital bits, are taken by an Android App. The computations performed through Fast Fourier Transformations (FFT) and Reaction and Movement time, enable the calculation of the severity of the PD symptoms, which results in an appropriate drug administration for that patient, at the moment when the measurement of patient finger pressures is taken. The novelty of this research is twofold. It allows a high level of personalization in PD treatment and uses modern technologies to bring new solutions in the field of drug administration to PD patients.
\end{abstract}

\section{Introduction}

The problem of drug administration for patients suffering from Parkinson's Disease (PD) has been known for decades and it has remained unresolved for many reasons. Firstly, successful treatments of PD symptoms depend heavily on a particular patient and his/her manifestation of PD symptoms, which is often associated with the individual experience a patient may have throughout the day. This means that we should personalize PD drug administration, in order to alleviate PD symptoms a patient may have at a particular moment. However, the personalization of medicine is still in its infancy. Its research currently focuses more on genome sequencing in order to learn how to group patients based on risk of disease, or response to therapy, or use diagnostic tests or techniques for each patient [1]. Secondly, we expect modern healthcare delivery to be supported by smart phones and numerous Apps we run on them. PD patients expect and possibly require, a new generation of hardware and software solutions in order to address their most urgent problem, which is personalized drug administration. Therefore in the second decade of the $21^{\text {st }}$ century we have an opportunity to look at new ways of resolving the problem of optimal treatments of PD symptoms using new technologies, which may bring new solutions to old problems. If we focus solely on the PD drug administration, then we should find a method of

(a) collecting information about PD symptoms a patient may have at any moment throughout the day

(b) advising the patient on the best possible drug administration at that moment when the information is collected.

Therefore, PD drug administration will vary from one moment to another throughout the day, because PD symptoms change. We should create a synergy of hardware and software solutions for performing (a) and (b), which enables the manipulation of sensor generated data, as the result of the measurements of the severity of PD symptoms. They are essential for appropriate drug administration. Creating an App which administers PD drugs, runs on patient smart phones, at the time when the measurement of the severity of PD symptoms is taken, would be the ultimate answer expected by PD patients and healthcare professionals.

This paper is organized as follows. In the next section we describe related work, and in section 3 we give a background of this research and explain the problems of PD drug administration. In section 4 we introduce prerequisite for developing

- ParMes device, attached to a patient smart phone which can measure the severity of PD symptoms,

- ParMesApp, an Android App, which takes these measurements and administers drugs accordingly.

These prerequisite include the way PD symptoms are estimated and drug administration calculated. In section 5 we explain the development of ParMes and ParMesApp, and evaluate our solution in the Conclusion. 


\section{Related Work}

At the time of writing this paper, we could not find any published work, which either personalizes PD drug administration through the measurement of the severity of PD symptoms, or offers a Software Architectural (SA) model for an App, which can advise on the best possible drug administration for a particular patient. Therefore we look at research in the field of remote patient monitoring and assisted living. One of the best sources, which compiles projects funded by the EU, is available at [2]. They focus on e-health [3] which changes the way we deliver health services and create modern healthcare environments. However, in [2] the authors divide e-health into comfort and security, rehabilitation and chronic disease telemonitoring. Our work should belong to the latter, because any persistent data generated within ParMesApp can be remotely accessed by any other e-health system and healthcare professionals. The comparative study from [2] pushes forward the problems, which have not been resolved yet, such as security in, impact of cognitive science on, and the existence of universal e-health systems.

The rapid changes and advances of mobile and wireless technologies, which have seriously impacted our everyday lives, have also infiltrated in healthcare $[4,5,6,7]$. We have witnessed the proliferation of wearable medical systems which have been paving the way towards pervasive and personalized healthcare $[8,9,10,11,12,13]$.

PD has also attracted the attention of various research groups in the domain of tele-monitoring and ehealth. There are numerous reports and peer reviewed papers which range from focusing on the optimization of symptomatic therapy for PD patients [14], techniques for diagnosing and monitoring PD patients [15] and projects which focus on wireless body area networks [16] to accurate telemonitoring of PD patients through speech tests [17], and medical devices which can be used in the managing and understanding degenerative diseases.

In terms of addressing the optimisation of PD treatment, we can find various approaches which range from the Tauberian approach [18], decision support tools for optimal PD drug administration as in [19], to "patient diaries", deep brain stimulation, and combining them with measuring/assessing levodopa-induced dyskinesia and bradykinesia in parkinsonian disorders [20,21].

The papers, which come slightly closer to our area of interest, study tremor frequency [22], measure rigidity of muscles [23], develop a quantitative method for 3D measurement of PD tremor, measure finger tapping contact force for quantitative diagnosis of PD, measure maximum pinching force to evaluate bradykinesia [24] and use single movement sensors for motor state detection and dyskinesia [25]. When looking at wearable sensors and systems for rehabilitation $[26,27]$ we can find works which use motion analysis [28] and lateral belt worn accelerometer [29].

The field of healthcare Apps designed for helping PD patients has been very poorly researched. We could find publications which monitor well-being through Apps [30], performing non invasive blood pressure measurement with Android phones, but there are no peer reviewed papers which focus solely on PD. The only publication which promotes a smart phone application for the detection of tremor is available at [31]. This application does not administer PD drugs.

\section{The Background: PD and Drug Administration}

PD is a degenerative disorder of the central nervous system [32,33,34]. It is a chronic and progressive movement disorder, with symptoms which worsen over time. Most common motor signs of PD are tremor, rigidity, bradykinesia and postural instability. Tremor usually occurs when a limb starts slightly shaking or oscillating with frequency $3-8 \mathrm{~Hz}$ [35]. Bradykinesia, or slow movement, is manifested when patients cannot perform fine tasks, they lose spontaneous movements and have difficulties with facial expressions [36]. Rigidity of muscles is common, uncomfortable and painful [37]. When the PD progresses, symptoms become more prevalent and increase the disability of patients.

PD is connected with death of nerve cells in substantia nigra, as a consequence of low production of dopamine and presence of Lewy bodies in remaining neurons. Lewy bodies are abnormal aggregates of protein that develop inside nerve cells and appear as spherical masses that displace other cell components [38]. Dopamine is a chemical that sends a message to the part of the brain for coordination and movement. When production of the dopamine drops below $80 \%$, doctors talk about PD and with over time, dopamine doesn't stop decreasing [39].

There is no cure for PD, but there are treatments which may alleviate the symptoms. The most frequently used is dopamine treatments [21], which replace missing dopamine with drugs such as Levodopa [40]. However, side effects are serious. PD patients experience dyskinesia or involuntary movements, vomiting, dizziness and hallucinations [39,21]. The severity of dyskinesia is related to the level of PD drug administration. When PD progresses and side effects of taking Levodopa become more severe, doctors choose Deep Brain Stimulation [41]. 


\subsection{The Problem with PD Drug Administration}

Optimization of PD treatment occurs when PD patients are administered the correct drug dosage, which suppress all symptoms, does not generate side effects and improve patient's wellbeing. However, PD symptoms vary from patient to patient, they depend on patient's activities and may change significantly throughout the day. It is extremely difficult to predict the exact drug dosage for any PD patient. They are often prescribed the same amount of drugs regardless of the current state of their symptoms [42]. This approach of suppressing PD symptoms develops side effects quicker and the treatment becomes ineffective $[43,44]$. The risk of dyskinesia is constant and depends on age, progress and severity of PD, dosage of drugs and the duration of Levodopa treatment.

In order to extend dopamine treatment and reduce Levodopa's side effects, many studies have been carried out towards the optimization of dosage of PD drugs. The amount of dopamine in the blood and PD state have negative correlation [22], which means the higher the state of PD, less dopamine is produced. In order to decrease PD's symptoms and increase the level of dopamine, with correct drug administration, scientists tried two different approaches. One is based on mathematical models for the drug administration [45] and the other on the tracking the progress of PD symptoms through patient diaries.

PD treatments based on patient diaries [46] initially showed promising results. They help, if a patient keeps his or her diary up to date. Doctors with diary's data may set up appropriate drug administration, change settings of the diaries $[47,48]$ and may prescribe treatments which will not bring new disabilities in PD patient's life. However, the drawback of this approach is well known. Doctors often find incomplete and constantly missing data in patient diaries, which often makes the optimisation of drug treatments unfeasible [49].

Administering drugs through predictions based on mathematical models, such as the Tauberan approach [18] or transfer function of second order [14], is based on the level of the concentration of the prescribed drug in the blood. However, PD symptoms vary from patient to patient and in this approach the specificity of PD symptoms, and ultimately personalisation of the drug administration are lost: all patients, with a particular level of the drug in the blood get the same dosage of medication, regardless of the severity of their PD symptoms.

Currently, there are no solutions which personalise PD treatments. In other words, there are no methods which can calculate the exact dosage for a particular PD patient at any time during the day.
In this research, we aim to address both: how to perform PD drug administration more efficiently and how to personalise it according to a particular patient's needs. Therefore the proposal is to build a device which can measure the status of tremor and bradykinesia in a PD patient and generate a software program which can calculate the most appropriate PD drug dosage for the patient. The solutions are in creating both: a device, equipped with sensors for the purpose of measuring PD symptoms and a software program, which manipulates sensor generated data in order to administer PD drugs. Our proposed solution is described in the following three sections.

\section{Prerequisite for Developing ParMes and ParMesApp}

We developed a ParMes device for measuring the severity of PD symptoms and ParMesApps Android App, which takes these measurements and calculates the best possible drug administration for a PD patient at the time when the measurements were taken. However, before we introduce both of them, we have to give a more detailed explanation on how the severity of PD symptoms can be estimated through ParMes and drug administration calculated, based on these estimates. In other words, understanding the role of estimation of tremor and bradykinesia, helps in explaining the design principles for creating both ParMess and ParMessApp.

\subsection{Estimating Symptoms of PD}

The severity of tremor and bradykinesia can be estimated by measuring the applied force on the screen of a smart phone. These two symptoms are visible on patient hands.

The level of dopamine and tremor frequency are in negative correlation [22]. When a patient has a low dopamine level, the power of tremor will be higher. The tremor's frequency occurs between 4-8 Hz. From obtained measures, which were taken for time period $\mathrm{T}$ and with sample frequency $256 \mathrm{~Hz}$, our software solution (ParMesApp) should calculate the Power Spectrum Density (PSD) [50] for every frequency in band pass between 4-8 Hz, and normalize these values.

Bradykinesia is measured with the help of applied pressure on the smart phone screen. From the obtained measurements, ParMesApp extracts reaction and movement time [24,14] and finds the highest value of applied pressure. Based on this value it calculates the:

- Reaction time (Eq. (1)) is measured as the time when stimulus changes its state and measured signal will reach threshold point which is $10 \%$ of the measured sample with the highest value. 
- Movement time (Eq. (2)) is measured as the time between the end of reaction time and when signal passes threshold $90 \%$ of the measured sample with highest value.

If we have $\mathrm{k}_{\mathrm{n}}$ ( $\mathrm{n}^{\text {th }}$ sample), $\mathrm{k}_{10 \%}$ (sample at $10 \%$ of maximum measured value), $\mathrm{k}_{90 \%}$ (sample at $90 \%$ of maximum measured value), $\Delta \mathrm{t}$ (time between two samples $\left(\Delta t=1 / f_{s}\right)$ and MMV - Maximum Measured Value, we can then define:

$$
\begin{gathered}
\mathrm{t}_{\mathrm{r}}=\mathrm{k}_{10 \%} \times \Delta \mathrm{t} \\
\mathrm{t}_{\mathrm{m}}=\left(\mathrm{k}_{90 \%} \times \Delta \mathrm{t}\right)-\mathrm{t}_{\mathrm{r}}
\end{gathered}
$$

\subsection{Calculating Drug Administration}

Even mathematics is used for the optimization of Levodopa treatment. In the pharmaceutical industry, producers of Levodopa developed the transfer function of the second order with drug administration as input. The output is the concentration Levodopa in blood, as given in [14]:

$$
G(s)=\frac{K}{(1+s T 1)(1+s T 2)}
$$

However, K, T1, T2 values were determined by measuring medicine concentration in blood, several times after the patient with PD was administrated $125 \mathrm{mg}$ of Levodopa. Because the benefits pf taking Levodopa appears 10-15 minutes after its administration, the authors of [14] added another variable in order to transfer function of the second order $e^{-s t}$ where $t$ is delay time. Therefore formula (4) replaces formula (3).

$$
G(s)=\frac{K e^{-s T}}{(1+s T 1)(1+s T 2)}
$$

This equation models pharmacodynamics of the drug and describes the relation of administrated drug and effect of the drug, i.e. its amount in the blood. Doctors predict that $1500 \mathrm{mg} / \mathrm{L}$ of the medicine in the blood is enough to suppress PD symptoms [52]. The level of medicine in the blood and the severity of the PD symptoms are in negative linear coloration and therefore we can calculate the difference between the current and desired amount of Levodopa in the blood. With this difference we can determine the required dosage of medicine, which won't harm the patient and will suppress symptoms.

The cost function of medicine usage is a solution for reducing the amount of administered medicine, where $\delta$ presents dosage of the medicine and $\mathrm{T}$ time between two sessions of drug administrations [51]:

$$
F(\Delta, T)=b \times \delta+\frac{C}{T}
$$

In order to reduce cost function $\mathrm{F}$, we have to decrease $\delta$ or increase T. $\delta$ is an impulse which determines the relation between medicine administration and plasma level in the blood and it is a response of a transfer function second-order system with time leg. The amplitude of the impulse is the amount of prescribed medicine. If we know the first applied dosage, which initiated the system, amplitude of impulse $\delta$ is the amount of the second dosage, the time response of the system will be:

$$
\mathrm{Y}(\mathrm{t})=40.89 \times \delta \times\left(\mathrm{e}^{-1.67(\mathrm{~T}-0.24)}-\mathrm{e}^{-18.57(\mathrm{~T}-0.27)}\right)
$$

However, however successful formulas (3)-(6) are, they do not take into account that PD patients have changeable need for Levodopa administration throughout the day. In other words we had to create a new formula because in our research we are interested in the amount of prescribe $\delta$ at any time in the day. From equation (6) we extracted $\delta$ and our final formula for administration of drug changes into;

$$
\delta=\frac{Y(t)}{40.89 \times\left(e^{-1.67(T-0.24)}-e^{-18.57(T-0.27)}\right.}
$$

$\mathrm{T}$ is determined as time between previous and current administration of the drug, while $Y(t)$ is the desired level of drug in the blood, based on the ratio of a symptom's severity of a PD patient and a healthy person in the same measurement range.

To summarise, we measure tremor and bradykinesia though the following:

Tremor: if the affected limb oscillates with a frequency between 4-8 Hz we estimate the severity of tremor on average PSD in bandpass from 4-8 Hz. The ratio is calculated from the average PSD of a PD patient and a healthy person and the desired level of drug in blood can be calculated as:

$$
\begin{gathered}
\mathrm{Y}_{\mathrm{t}}(\mathrm{t})=1500 \mathrm{mg} / \mathrm{L} \times(\text { avrPSDPD patient } / \\
\text { avrPSD } \left._{\text {healty person }}\right)
\end{gathered}
$$

Bradykinesia: the desired level of drug in the blood for patients with Bradykinesia is calculated upon reaction and movement time. We summarised reaction (Eq. 1) and movement (Eq. 2) time of a PD patient and a healthy person. Based on summation and ratio between summation, we calculated the desired level of drug as: 
$\mathrm{Y}_{\mathrm{t}}(\mathrm{t})=1500 \mathrm{mg} / \mathrm{L} \times$

((reaction+movement time) PD patient

/(reaction+movement time) $)_{\text {healty person })}$

\section{ParMes Device}

The main purpose of ParMes is to measure applied pressure of a human finger on its screen.

For creating ParMes we had two options. We could either attach ParMes on the back of a smart phone, in which case ParMes becomes a "slave", or create a completely new device which would not need a smart phone and would perform all: measurements, drug dosage calculation and interaction with a PD patient.

Option 1 allows the use of parts and existing functionalities of smart phones and takes advantage of numerous software solutions, which exist on smart phones, in order to perform computations and ultimately deliver services expected by a PD patient.

Option 2 would require more resources in order to deliver both: creation of hardware parts for ParMes (including screens) and software components. This option can not offer as attractive operating environments for software development as our smart phones do.

We chose option 1 and "the screen", which we refer to is the screen of a smart phone. Our ParMes should behave as a slave device. When ParMes receives a request to measure a pressure caused by a PD patient's finger, it starts measuring the applied force on the screen and sends the results of the measuring to the smart phone. Therefore, smart phones manipulate the measurements of pressure caused by a human finger.

There were two important issues which had to be addressed before ParMes was designed.

Firstly, we had to take into account that the time between the beginning and the end of the measurement of a pressure, caused by a human finger, with ParMes is 10 seconds. In this period ParMes sends 256 samples of applied force per second.

Secondly, we had to design hardware, which will meet special requirements. It is supposed to be small and convenient to use.

ParMes includes some basic analogue components such as an operational amplifier, switches and elements with resistive and capacitive properties and digital logical operators such as AND, OR, NOR, NAND, XOR, MUX and D-FlipFlops. They will naturally convert into electrical circuits. Therefore ParMes has Analogue part, which measures voltage resulting after applying finger pressure and Digital part, which transforms these measurements into digital streams.
Picture 1 shows the PCD hardware design of ParMes and Figure 2 shows how its device is attached to a smart phone.

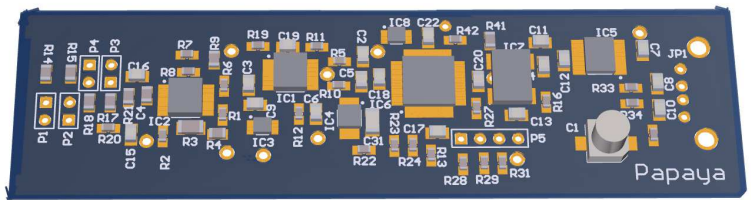

Figure 1. РСB hardware design of ParMes

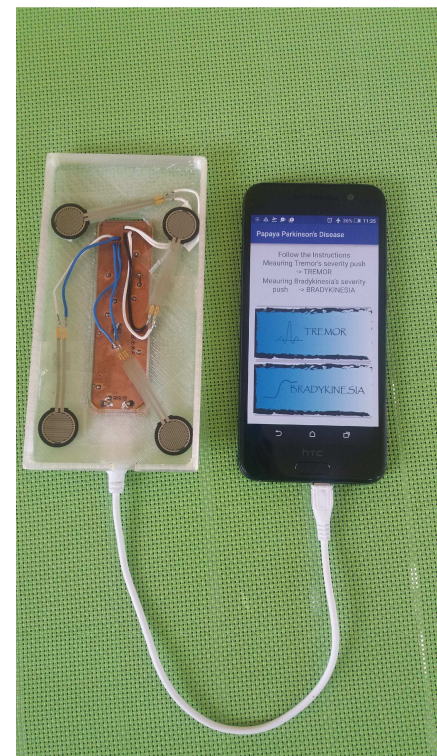

Figure 2. ParMes device attached on smart phone

\section{ParMesApp Android Application}

The design and development of the ParMesApp have three prerequisites.

Firstly, an overall SA was needed for creating an environment where ParMesApp can reside and use sensor generated data by ParMes. The SA must also accommodate the functionality, which is expected to be delivered through the ParMesApp. However, in this highly interactive and mobile environment, User Interfaces (UI) usually govern the logic of the manipulation of ParMesApp by a particular PD patient. Consequently, the functionalities depicted in the SA must be accompanied by a set of UI, which illustrate the way our ParMesApp works and the way a PD patient may manipulate it. The UI for our ParMesApp is given in Figure 3. 
Secondly, the abstract model of the SA, must be created through either UML diagrams or software patterns like Model-View-Controller (MVC) [52]. It should be layered and component based. It should also deliver a generic solution for the manipulation of sensor generated data from ParMes and consequently absorb or accommodate all possible changes we may have in future when using ParMesApp. The SA should also accommodate the way the calculations of Tremor/ Bradykinesia/PD drug administration are performed: we should be able to change the code for calculations and algorithms in future, if required.

Thirdly, software components defined through the ParMesApp SA must be deployable within the Android Integrated Development Environment (IDE), such as Android Studio. Therefore, abstract SA components become technology specific in order to have a successful deployment of ParMesApp. It is assumed, particularly if MVC pattern is used, that SA strictly distinguishes between UI defined at the front end of ParMesApp and repositories of data, generated by sensors within ParMes, which are used for calculating drug administration for PD patients.

Figure 3 shows UIs for ParMessApp. In the first UI, only two buttons are available: "TREMOR" and "BRADYKINESIA". When a PD patient chooses which type of measurement he/she needs, the ParMesApp will lead the patient through the measurement procedure. When the measurement is completed, ParMesApp will show the results which are expected to be "Severity of PD symptoms" and "Recommended drug administration". The result screens are visible in the lower part of Figure 3.

Figure 4. is a generic, layered and component based SA model for ParMesApp. It uses the Model-ViewController (MVC) pattern. This model is deployable in many component based operating environments which are underpinned with component technologies [53,54]. Android IDE uses plug-ins which deploy the architectural solution from Figure 4. within the Android Studio.

In Figure 4, UIs, which are illustrated in Figure 3, are in boxes with yellow borders. Computation which control the ParMesApp are in red, calculations of drug dosage (named FFT and R\&M) are in green and data repositories are in blue. A strict division between UI, computations and data is essential. This makes the SA solution from Figure 4 reusable in any other IDE.

ParMesApp may have two different types of repositories. One should be a pool of sensor-generated data (SGD) by ParMes, which contains measurement of a PD patient's finger pressure. This data does not have to be persistent. SGD belong to a particular moment of measurement and cannot be used again for a new calculation of the drug dosage. The other type of data is a traditional database repository, i.e. persistent data, which may store demographic and clinical data relevant to a particular PD patient. The development of the latter is outside the scope of this paper. However, access to persistent data must be guaranteed through the SA offered for ParMesApp. In other words, the SA model gives provision for accessing any persistent repository of structured data, if needed.

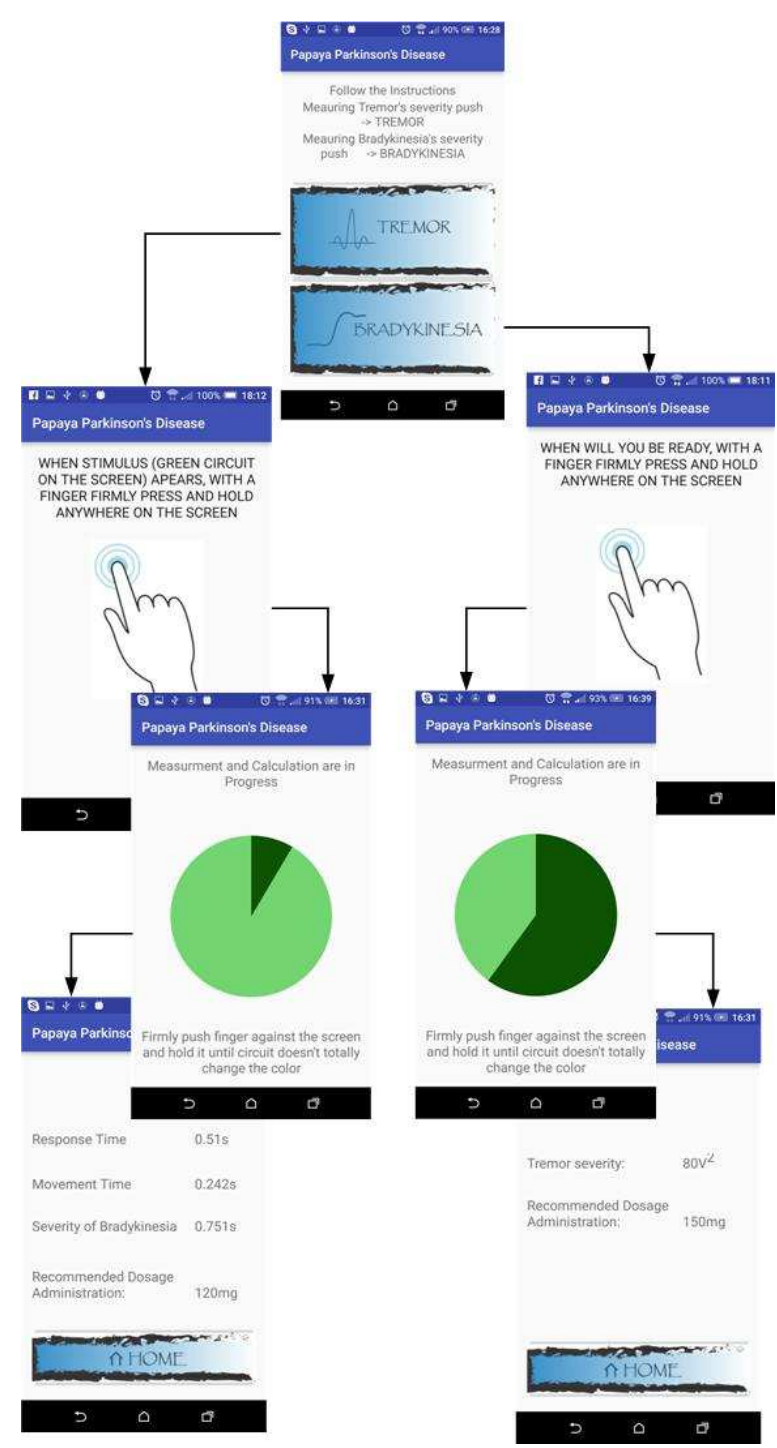

Figure 3. User interfaces in ParMesApp

It is important to note that all computations in Figure 4, which need data, will be performed after retrieving relevant data. This applies to all types of data: it can either be sensor generated data from ParMes or persistently stored data, about PD patients. This puts forward the main characteristics of this SA solution: all 
components shown in Figure 4 might be located on different nodes of any computer network. The solution consequently allows data sharing in their repositories and makes provisions for flexibility in case we change the way we digitalize analogue signals of ParMes, or change the way we calculate recommended drug dosage. This is particularly important if advances in research on treatments of $\mathrm{PD}$ would make the change necessary.

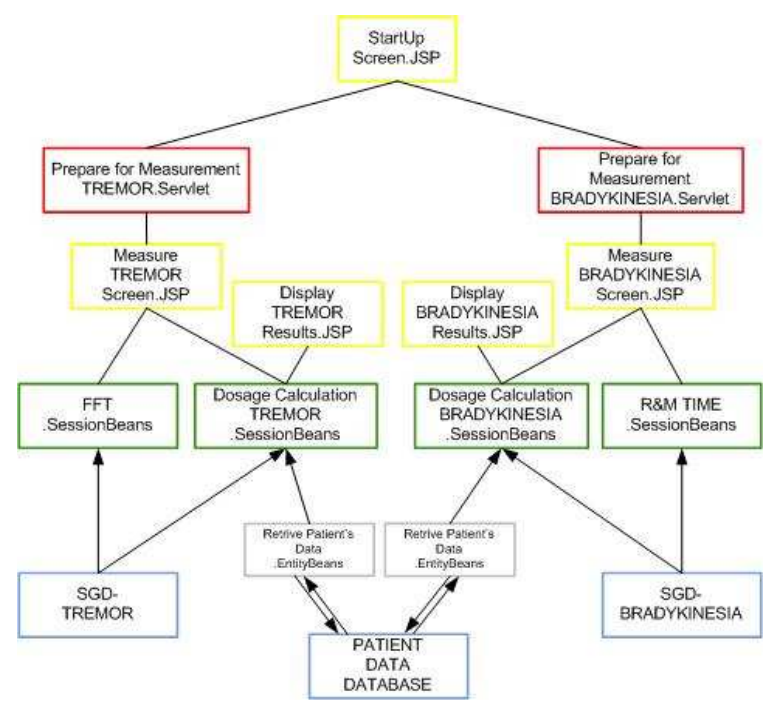

Figure 4. Software Architecture for ParMesApp

Finally, sensor generated data may not be important to store and keep it as historical data, i.e. it might not be advisable to make it persistent. What is important here is to make decision on the PD drug administration possibly persistent and encourage frequent measurements of Tremor and Bradykinesia in order to minimize errors when administering PD drugs.

\section{Conclusions}

In this paper we demonstrate that it is feasible to utilise modern hardware/software technologies for creating dynamic cyber-physical environments where PD patients are able to measure the severity of their symptoms, at any time throughout the day, and have the PD drugs administered according to these measurements. ParMes and ParMesApp have been developed as prototypes, but their commercialisation is a small step away from the results of this research.

There are two important outcomes from this research.

The evaluation of ParMes and ParmessApp has revealed that the idea of creating a device, attaching it to a smart phone and running an App on the phone, in order to measure the rigidity of PD patient's muscles, is reusable in other healthcare domains. This was not expected. Both solutions ParMes and ParmessApp will require no major changes if we wish to use both in different environments and for different purposes.

However, the evaluation prompted another important aspect of ParMes, which should be debated. While testing ParMes and analysing Apps available on our smart phones, it became obvious that smart phones are becoming more efficient in managing our wellbeing and more popular in the delivery of healthcare, then various gadgets specially designed for healthcare. Therefore we had to ask questions: Was our PaerMes necessary? Would we achieve the same result if we used either Android or iOS devices and run ParMessApp on them?

We debate these two outcomes in section 7.1 and 7.2 .

\subsection{Evaluation}

It is important to note that connecting ParMes, equipped with sensors which generate data, with an Android App, which is built upon a specific SA style from Figure 4, answers many questions we may have when evaluating the results of this research.

Firstly, the generic SA style from Figure 4, based on MVC pattern, secures the flexibility of the ParMesApp because its computational components are separated from persistence / data repositories and UI. Any future changes we may have when estimating tremor and bradykinesia and calculating the recommended drug dosage can be easily accommodated within the ParMesApp, without affecting most of its software components.

Secondly, if we needed readings of anyone's finger pressure on a smart phone screen for any other condition or illness, the SA of ParMesApp provides a clearly defined UI and repository components which can be developed for drug administration not related to PD. In other words, we can use the model defined in Figure 4 for drug administration of any other condition which may depend on the measurements of someone's finger pressures.

Thirdly, the SA from Figure 4 is a sound way of creating Apps if we wish them to be robust and reliable when they are run by patients. If the SA style defined in Figure 4 is deployable within an Android Studio, as an IDE, then we should be able to promote this particular way of constructing software for pervasive healthcare. In other words, any future App which depends on the manipulation of sensor generated data, should exploit the power of Figure 4 before developers 
start coding in Android or any other mobile /wireless operating environments.

Finally, Our ParMesApp delivers advice on PD drug administration at the moment when the measurement of PD symptoms are taken. It personalizes drug administration and makes it precise to fit the needs of a patient, at the moment when the patient decides. ParMesApp can run many times per day, when PD symptoms change. This might be the most important outcome of this research.

\subsection{ParMes in Testing}

Throughout the development process of ParMes and ParMesApp we were constantly aware that our idea of creating a separate mobile device ParMes for the measurements of the finger pressure of a PD patient may lead us towards a completely different debate on the efficiency of having two mobile devices.

Our ParMes is attached to a smart phone and in numerous testing, this synergy worked very well. We proved that it was not difficult to design and develop ParMes, but it was extremely difficult to place the computations for drug administration on it. We needed a smart phone and its operating environment, if we wished to manipulate sensor-generated data. Smart phones give much more power and opportunities for creating intelligent software solutions, compared to any device (gadet) equipped with sensors.

Consequently having a new developed gadget ParMes as a "slave" to any Android smart phone, in order to enable decision making on PD drug administration, was a necessity. Patients do want to run Apps on their own smart phones and therefore there was no other way but to use ParMes as a slave. We could not make conclusions on the readiness of smart phone technologies to replace our ParMes, but the computational intelligence we could create on ParMes could not be compared with powerful Android, which is present almost everywhere and across environments where we need to run Apps.

\subsection{Future Work}

Considering our debate in sections 7.1. and 7.2, more research is needed to investigate how smart phones might replace ParMes in future. Computationally powerful Android environments can carry sophisticated calculations, which could question the existence of numerous gadgets we buy off the shelf. These gadgets assist in our everyday lives, but do not have enough of their own power in order to create intelligent software solutions for users.

It has to be debated if the future of pervasive healthcare is in (a) creating more mobile devices equipped with sensors, and add more computational power to them or

(b) increasing the use of smart phones, instead of developing more gadgets equipped with sensors. Smart phones provide a window of opportunities because they live in Android (or any other similar) operational environments and can create naturally more intelligent software solutions for the delivery of healthcare.

Our ParMesApp on an Android phone can manipulate sensor-generated data and perform relatively complicated calculations, but ParMes alone can not do it. It remains to be seen which way gadget manufacturers and App developers will go. Whatever the future of ParMes would be, the reusability of SA form Figure 4 guarantees successful creation of Apps in any operating environment, if relevant sensor generated data is supplied.

\section{References}

[1] "Recent Developments in Personalized Medicine", by The Royal Society, London, UK , 2015, available at https://royalsociety.org/ /media/policy/Publications/2015/per sonalised-medicine-report.pdf

[2] O. Hamdi, M. A. Chalouf, and D. Ouattara, "eHealth : Survey on research projects , comparative study of telemonitoring architectures and main issues," Netw. Comput. Appl., vol. 46, pp. 100-112, 2014.

[3] J. P. Harrison and A. Lee, "The Role of E-Health in the Changing Health Care," Nurs. Econ., vol. 24, no. 6, pp. $283-$ $288,2006$.

[4] Y. Akematsu and M. Tsuji, "Economic Effect of eHealth : Focusing on the Reduction of Days Spent for Treatment," IEEE, pp. 14-20, 2009.

[5] G. Graschev, S. Rakowsky, T.A. Roelofs, P.M. Schlag, "New Medicla Technologies of Future", in Proceedings of the Internaitonal Conference on Digital Technologies (DT), IEEE, pp 84-89, 2013.

[6] C. Vandelanotte, C. E. Short, M. M. Andre, M. Hingle, N. Nathan, B. Hons, S. L. Williams, M. L. Lopez, S. Parekh, and C. A. Maher, "Report Past, Present, and Future of eHealth and mHealth Research to Improve Physical Activity and Dietary Behaviors," Journal Nutrition, Education and Behaviour, vol. 48, no. 3, pp. 219-228, March 2016.

[7] E. Reilent, I. Loobas, R. Pahtma, A. Kuusik, "Medical and Contetx Data Acquisition System for Paitent Home Monitoring", in Proceedings of the $12^{\text {th }}$ Biennial Baltic Eøectronics Conference (BEC 2010), Tallin, Esotnia, pp. 269-272, 2011.

[8] X.-F. Teng, Y.-T. Zhang, C. C. Y. Poon, and P. Bonato, "Wearable Medical Systems for p-Health," IEEE Review on. Biomedical Engineering., vol. 1, pp. 62 - 74, 2008. 
[9] A. Lymberis, "Smart wearable systems for personalised health management: Current R\&D and future challenges," Proc. 25th Ann. Int. Conf. IEEE EMBS, vol. 4, pp. 37163719, 2003.

[10] G. TrÃster, "The agenda of wearable healthcare," IMIA Year Book of Medical Informatics 2005 Ubiquitous Health. Care Systems (Haux R, Kulikowski C, editors) pp. 125-138, 2004.

[11] J. E. Mezzich, "Psychiatry for the Person: articulating medicine's science and humanism" World Psychiatry, vol. 6, no. 2, pp. 65-67, 2007.

[12] P.B. Shull, D. Damian "Haptic wearables as sensory Replacement, snesory augmentation and trainer - a review", in Journal of NeuroEngineering and Rehabilitaiton, 12(59), 2015.

[13] R. Shojanoori, R. Juric, R., M. Lohi, "Computationally Significant Semantics in Pervasive Healthcare", Journal of Integrated Design and Process Science, IOS Press, Volume 16, Number 1-2012, pp 43-62. 2012.

[14] S.S. Hacisalihzade, M. Mansour, and C. Albani, "Optimization of symptomatic therapy in Parkinson's disease.," IEEE Trans. Biomed. Eng., vol. 36, no. 3, pp. 363372, 1989.

[15] R. Prasad, S.S. Babu, N. Siddalah, K.S. Rao "A Review of Techniques for Diagnosing and Monitoring Paitents with Parkinson Disease", in Journal of Biosensors and Bioelectronics, Volume 7, Number 1, 2016.

[16] M. Sarestonemi, J. Iinati, N. Keranen, T. Jamsa, J Partala, T. Seppanen, J. Reponen, J. "Receiver Prformance on IEEE 802.15.6 based WBAN for Monitoring Parkinson Disease", in Proceedings of the 8th International Symposium on Medical Information and Communication Technology (ISMICT), 2014.

[17] Tsanas, A., Little, M.A., McSharry, P., Ramig, L. "Accurate telemonitoring of Parkinson disease progression by non-invasive speech tests", IEEE Transacitons on Biomedical Engineering, 2010, 57(4)

[18] S. S. Hacisalihzade, M. F. Senning, and R. Strotz, "Optimization of Drug Administration by a Tauberan Approach," IEEE Transacitons on Biomedical Engineering, vol. 34, issue 4, pp. 430-436, 1987.

[19] M. G. Tsipourasa, A. T. Tzallasa, G. Rigasa, S. Tsoulib, and Dimitrios I. Fotiadisa Spiros Konitsiotis, "An automated methodology for levodopa-induced dyskinesia: Assessment based on gyroscope and accelerometer signals," Artif. Intell. Med., vol. 55, no. 2, pp. 127-135, 2012.

[20] G. Pal and C. G. Goetz, "Assessing bradykinesia in Parkinsonian disorders," Front. Neurol., vol. 4, no. June, pp. $1-5,2013$.
[21] B. Thanvi, N. Lo, and T. Robinson, "Levodopa-induced dyskinesia in Parkinson's disease: clinical features, pathogenesis, prevention and treatment," Postgrad. Med. J., vol. 83, no. 980, pp. 384-388, 2007.

[22] Y. Matsumoto, K. Shindo, M. Sakakibara, M. Tamura, and I. Fukumoto, "Study on the $8 \mathrm{~Hz}$ tremor frequency related to dopamine level in Parkinsonian patients," Proceedings of the Annual. Inernational Conference IEEE Eng. Med. Biol., vol. 4, pp. 1771-1772, 1996.

[23] A. Prochazka, D. J. Bennett, M. J. Stephens, S. K. Patrick, R. Sears-duru, T. Roberts, and J. H. Jhamandas, "Measurement of Rigidity in Parkinson' s Disease," Mov. Disord. Soc., vol. 12, no. 1, pp. 24-32, 1997.

[24] T. Touge, K. Tsutsui, K. Kume, K. Ikeda, K. Deguchi, Y. Nakamura, H. Sciences, and S. Hospital, "Measuring the Maximum Pinching Force is a Good Procedure to Evaluate Bradykinesia in Patients with Parkinson's Disease," Complex Med. Eng. (CME), 2011, pp. 192-194, 2013.

[25] Sama, A., Perez-Lopez, C., Romagosa, J. RodriguezMartin, D., Catala, A., Cebastany, J., Perez-Martinez, D.A., Rodriguez-Molinero, A. "Dyskinesia and motor state detection in Parkinson's disease patients with a single movement sensor", Porceedings of the $34^{\text {th }}$ Annual Conference of the IEEE EBMS, pp 1194-1197, 2012.

[26] S. Patel, H. Park, P. Bonato, L. Chan, and M. Rodgers, "A review of wearable sensors and systems with application in rehabilitation," in Journal of Neuroengineering Rehabilitaions, Vol. 9, no. 21, pp. 1-17, 2012.

[27] P. Bonato "Wearable Sensors and Systems", in IEEE Engineering in Medicine and Biology Magazine, vol. 29, no. 3, pp. 25-36, 2010.

[28] G. Lo, A.R. Suresh, L. Stocco, S. Gonzales-Valenzuela, V.C.M. Leung, "A Wireless Sensor System fo rmOtion Analysis of Parkinson's Disease Paitents", delivered at PerCom workshop in 2011 as work in Progress.

[29] Sayeed, T., Sama, A., Catala, A., Cebastany, J. "Comparative and adaptation of step detection and step length estimators to lateral belt worn accelerometer", in IEEE HEALTHCOM, The $1^{\text {st }}$ internaitonal Workshop on Service Science for e-Heaølth, 2013.

[30] E. Lane, M. T. Willams, T. Olds, and C. Maher, "An internet-based physical activity intervention for adolescents with cerebral palsy: a randomized controlled trial.," Dev Med Child Neurol, vol. 52, no. 5, pp. 448-55, 2010.

[31] V. Parra, P. Milano, G. Figueras, M. Huerta, I. Member, A. Marzinotto, R. Gonzalez, and R. Alvizu, "A Smartphone Application for Parkinson Tremor Detection”, VI Latin American Congress on Biomedical Engineering CLAIB 2014, Paraná, Argentina, Volume 49 of the series IFMBE Proceedings pp 785-788, 2015. 
[32] J. Parkinson, “An essay on the shaking palsy. 1817.," J. Neuropsychiatry Clin. Neurosci., vol. 14, no. 2, pp. 223-236; discussion 222, 1817.

[33] S. D. Kim, N. E. Allen, C. G. Canning, and V. S. C. Fung, "Postural instability in patients with Parkinson's Disease: Epidemiology, pathophysiology and management," CNS Drugs, vol. 27, no. 2, pp. 97-112, 2013.

[34] B. Thomas and M. F. Beal, "Molecular insights into Parkinson's disease", F1000 Medical Report, vol. 16, no. 2, pp. 183-194, 2007.

[35] T. R. Mhyre, J. T. Boyd, R. W. Hamill, and K. A. Maguire-Zeiss, "Protein Aggregation and Fibrillogenesis in Cerebral and Systemic Amyloid Disease," Subcell. Biochem. vol. 65, pp. 389-455, 2012.

[36] J.C. Berardelli, J. C. Rothwell, P. D. Thompson, and M. Hallett, "Pathophysiology of bradykinesia in Parkinson's disease.," Brain, vol. 124, no. Pt 11, pp. 2131-2146, 2001.

[37] B. Sepehri, G. A. Shahidi, A. Esteki, and M. Moinodin, "Measurement of rigidity in elbow joint an objective method for evaluation of rigidity involved diseases," ICBPE 2006 Proc. 2006 Int. Conf. Biomed. Pharm. Eng., p. a -f, 2006.

[38] Dickson, Feany, Yen, Mattiace, and Davies, "Cytoskeletal pathology in non-Alzheimer degenerative dementia: new lesions in diffuse Lewy body disease, Pick's disease, and corticobasal degeneration," J Neural Transm Suppl., vol. 47, pp. 31-46, 1996.

[39] M. M. Goldenberg, "Medical management of Parkinson's disease.," P T Journal, vol. 33, no. 10, pp. 590606, 2008.

[40] S. L. Smith, M. A. Lones, M. Bedder, J. E. Alty, J. Cosgrove, R. J. Maguire, M. E. Pownall, D. Ivanoiu, C. Lyle, A. Cording, and C. J. H. Elliott, "Computational approaches for understanding the diagnosis and treatment of Parkinson's disease," IET Syst. Biol., vol. 9, pp. 1-8, 2015.

[41] S. Patel, T. Hester, R. Hughes, N. Huggins, D. Standaert, A. Flaherty, and P. Bonato, "Using wearable sensors to enhance DBS parameter adjustment for Parkinson's disease patients through measures of motor response," Proc. 3rd IEEE-EMBS Int. Summer Sch. Symp. Med. Devices Biosensors, ISSS-MDBS 2006, pp. 141-144, 2006'

[42] J. Jankovic, "Current approaches to the treatment of Parkinson' s disease," Neuropsychiatric Disease and Treatment, vol. 4, no. 4, pp. 743-757, 2008.

[43] C. S. Okereke, L. Kirby, D. Kumar, E. I. Cullen, R. D. Pratt, and W. A. Hahne, "Concurrent administration of donepezil $\mathrm{HCl}$ and levodopa / carbidopa in patients with Parkinson' s disease : assessment of pharmacokinetic changes and safety following multiple oral doses," $B r . J$.
Clin. Pharmacol., vol. 41, pp. 41-49, 2004

[44] M. Yokochi, "Reevaluation of levodopa therapy for the treatment of advanced Parkinson's disease," Park. Realt. Disord., vol. 15, pp. S25-S30, 2009.

[45] J. Best, G. Oakley, M. Reed, and H. F. Nijhout, "Mathematical models: interactions between serotonin and dopamine in Parkinson's Disease.," Etiol. Pathophysiol. Park. Dis., pp. 405-420, 2011.

[46] N. Saito, T. Yamamoto, Y. Sugiura, S. Shimizu, and M. Shimizu, "Lifecorder: a new device for the long-term monitoring of motor activities for Parkinson's disease.," Intern. Med., vol. 43, no. 8, pp. 685-692, 2004.

[47] J. Schneider, D. Novak, and R. Jech, "Optimization of Parkinson Disease treatment combining anti-Parkinson drugs and deep brain stimulation using patient diaries.," Conf. Proc. ... Annu. Int. Conf. IEEE Eng. Med. Biol. Soc. IEEE Eng. Med. Biol. Soc. Annu. Conf., vol. 2015, pp. 3444-7, 2015.

[48] O. Rascol, D. J. Brooks, E. Melamed, W. Oertel, W. Poewe, F. Stocchi, and E. Tolosa, "Rasagiline as an adjunct to levodopa in patients with Parkinson's disease and motor fluctuations (LARGO, Lasting effect in Adjunct therapy with Rasagiline Given Once daily, study): A randomised, doubleblind, parallel-group trial," Lancet, vol. 365, no. 9463, pp. 947-954, 2005.

[49] J. Reimer, M. Grabowski, O. Lindvall, and P. Hagell, "Use and interpretation of on/off diaries in Parkinson's disease," J Neurol Neurosurg Psychiatry, vol. 75, pp. 396400, 2004.

[50] T. Heida, E. C. Wentink, and E. Marani, "Power spectral density analysis of physiological, rest and action tremor in Parkinson's disease patients treated with deep brain stimulation.," J. Neuroeng. Rehabil., vol. 10, no. 1, p. 70, 2013.

[51] M. Haeri, Y. Sarbaz, and S. Gharibzadeh, "Modeling the Parkinson's tremor and its treatments," J. Theor. Biol., vol. 236, no. 3, pp. 311-322, 2005.

[52] E. Gamma, R. Helm, R. Johnson, J. Vlissides "Design Patterns: Elements of Reusable Object-Oriented Software, Addison Wesley 1995.

[53] N. E. T. Framework, , "Overview of the . NET Framework," pp. 1-4, 2016.

[54] E. J. Developer and G. Release, "Oracle9 i AS Containers for J2EE,” vol. 2, no. August, 2002. 